\title{
A reversible full adder using adiabatic superconductor logic
}

Taiki Yamae $^{1}$, Naoki Takeuchi ${ }^{2,3}$, and Nobuyuki Yoshikawa ${ }^{1,2}$

${ }^{1}$ Department of Electrical and Computer Engineering, Yokohama National University, 79-5

Tokiwadai, Hodogaya, Yokohama 240-8501, Japan

${ }^{2}$ Institute of Advanced Sciences, Yokohama National University, 79-5 Tokiwadai, Hodogaya, Yokohama 240-8501, Japan

3 PRESTO, Japan Science and Technology Agency, 4-1-8 Honcho, Kawaguchi, Saitama 332-0012, Japan

E-mail: yamae-taiki-zd@ynu.jp

Abstract. Reversible logic circuits can perform logic operations in a thermodynamically reversible manner, or without energy dissipation. The reversible quantum-flux-parametron (RQFP) is a reversible logic gate using adiabatic superconductor logic. In the present study, we design and demonstrate a reversible full adder (RFA) using RQFP gates in order to demonstrate that RQFP gates can be used as building blocks to design reversible logic circuits. An analysis of the time evolution of the phase differences across the Josephson junctions in the RFA showed that its logic state can change quasi-statically during a logic operation. Calculation of the energy dissipation of the RFA showed that it decreases in proportion to the operating frequency. These numerical calculation results ensure that the RFA is thermodynamically and logically reversible. In addition, we experimentally demonstrated correct operation of the RFA for all input data combinations. These results reveal that logic circuits designed using RQFP gates can perform reversible computing, i.e., RQFP gates can be used as building blocks of reversible logic circuits. 


\section{Introduction}

For several decades, complementary metal-oxide-semiconductor (CMOS) logic has been primarily used in large-scale integrated circuits, thereby driving the advancement of information technologies. The performance and energy efficiency of CMOS logic has been improved by reducing the physical size of CMOS devices. However, the miniaturization of CMOS devices is expected to shortly reach physical limitations. The power consumption of an exascale supercomputer using CMOS logic is estimated to exceed $100 \mathrm{MW}[1,2]$, even if we assume continuous improvement of the CMOS technology. Therefore, very energy-efficient logic devices are required in order to achieve future high-end computers. Reversible computing [3, 4], the concept of which is different from conventional CMOS-based logic, is attractive for increasing the energy efficiency of logic circuits because the energy dissipation of logic circuits can be reduced in proportion to the operating frequency. Thus far, several reversible logic gates have been proposed using different devices, including superconducting devices [5], magnetic devices [6], quantum-dot cellular automata [7], nanomechanical devices [8], and enzyme-based biocatalytic systems [9].

In a previous study, we proposed the reversible quantum-flux-parametron (RQFP) [10], which is a logically and thermodynamically reversible logic gate that uses the adiabatic quantum-flux-parametron (AQFP) $[11,12]$. AQFP logic is an adiabatic superconductor logic that is based on the quantum-flux-parametron (QFP) [13], which was proposed by Eiichi Goto. Numerical simulations [14] suggested that RQFP gates can perform logic operations in a thermodynamically reversible manner, or without energy dissipation, if RQFP gates are operated quasi-statically. Furthermore, we fabricated an RQFP gate using the $\mathrm{Nb}$ integrated-circuit fabrication process [15] provided by the National Institute of Advanced Industrial Science and Technology (AIST), and demonstrated its correct logic operations at liquid He temperature [16]. 
An advantage of RQFP gates is that these gates can be used as building blocks for large-scale reversible logic circuits because any combinational logic can be formed using RQFP gates, as will be shown later. However, it remains unclear whether circuits composed of multiple RQFP gates are thermodynamically reversible, as is the case for single RQFP gates. In addition, reversible logic circuits composed of multiple RQFP gates have yet to be experimentally demonstrated. In the present study, we designed a reversible full adder (RFA) using RQFP gates to investigate the reversibility and feasibility of RQFP-based logic circuits. We analyzed the time evolution of the phase differences across the Josephson junctions in the RFA and calculated its energy dissipation to demonstrate thermodynamic reversibility. We also fabricated an RFA and examined its operation to reveal that RQFP-based logic circuits can operate stably using practical circuit parameters and fabrication technology.

\section{Reversible QFP}

Figure 1 shows a schematic of the RQFP gate, which includes six symmetrically interconnected AQFP gates. The circuit parameters are based on a previous study $[14,15]$. Gates A through $\mathrm{C}$ work as three-output splitter (SPL) gates, which are buffer gates with a fan-out of three. Gates X through $\mathrm{Z}$ work as three-input majority (MAJ) gates, the logic states of which are determined by the majority vote of the inputs: $\operatorname{MAJ}(i, j, k)=(i \wedge j) \vee(j \wedge k) \vee(k \wedge i)$, where $i, j$, and $k$ are the binary inputs. Using this MAJ function, the logic state of gate $\mathrm{X}$ is given by $x=$ $\operatorname{MAJ}(\neg a, b, c)$ because gate $\mathrm{X}$ receives $\neg a, b$, and $c$ from gates $\mathrm{A}, \mathrm{B}$, and $\mathrm{C}$, respectively. Similarly, the logic states of gates $\mathrm{Y}$ and $\mathrm{Z}$ are given by $y=\operatorname{MAJ}(a, \neg b, c)$ and $z=\operatorname{MAJ}(a, b, \neg c)$, respectively. Therefore, the RQFP gate performs the following operation:

$$
(x, y, z)=(\operatorname{MAJ}(\bar{a}, b, c), \operatorname{MAJ}(a, \bar{b}, c), \operatorname{MAJ}(a, b, \bar{c}))
$$

Note that logical negation is achieved by inverting the sign of the coupling coefficients $k_{\text {sout }}$, 


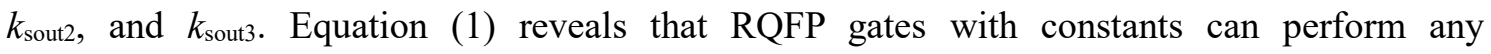
combinational logic. For instance, an AND operation of $a$ and $c$ is performed by applying 1 to $b$ : $y=a c$ for $b=1$. Then, $I_{\mathrm{x} 1}$ and $I_{\mathrm{x} 2}$ are ac excitation currents that apply an ac magnetic flux to the AQFP gates in the RQFP gate so that the AQFP gates are powered and clocked, and $I_{\mathrm{d}}$ is a dc-offset current that applies a magnetic flux of $0.5 \Phi_{0}$ to each AQFP gate, where $\Phi_{0}$ is a flux quantum. When $I_{\mathrm{x} 1}\left(I_{\mathrm{x} 2}\right)$ is applied, a SPL (MAJ) gate generates an output current $I_{\text {sout }}\left(I_{\mathrm{mout}}\right)$, the sign of which represents the logic state. A positive (negative) output current corresponds to a logic state of $1(0)$. The output currents apply a magnetic flux to the next gates via transformers. The details of the operating principles and excitation methods of AQFP logic are given in the literature $[11,12,15]$. The truth table for RQFP gates clearly indicates that the gates are bijective, or logically reversible [10]. Moreover, RQFP gates can operate thermodynamically reversibly because [14]: (i) the state variables of RQFP gates can evolve quasi-statically, or time reversibly, during a logic operation, and (ii) the energy dissipation required for a logic operation performed by an RQFP gate approaches zero as the operating frequency decreases. Therefore, RQFP gates are thermodynamically, as well as logically, reversible.

\section{Reversible full adder}

\subsection{Operation principle}

Figure 2 shows a schematic of the RFA, which is composed of two RQFP gates, where RQFP1 includes gates A1 through Z1 and RQFP2 includes gates A2 through Z2. Although the locations and numbers of logic negation in RQFP1 and RQFP2 are different from those of the original RQFP gate shown in figure 1, RQFP1 and RQFP2 are also logically reversible and can operate thermodynamically reversibly, as will be shown later. The number of Josephson junctions used in the RFA is 28 . Here, $a, b$, and $c_{\text {in }}$ are the inputs, and $s$ and $c_{\text {out }}$ are the sum and carry outputs, 
respectively. In addition, $x$ and $y$ correspond to $c_{\text {in }}$ and $b$, respectively, and are regarded as garbage outputs [3], which are required in order to ensure logical and thermodynamic reversibility. Table 1 shows the truth table for the RFA, which is bijective, or logically reversible [17]. Figure 3 shows the transient analysis results for the RFA using the Josephson circuit simulator, JSIM [18]. In the figure, $I_{\mathrm{x} 1}$ and $I_{\mathrm{x} 2}$ are $1 \mathrm{GHz}$ excitation currents. In addition, $I_{\text {outA } 1}$ through $I_{\text {outZ1 }}$ are the output currents for each gate in RQFP1, and $I_{\text {outA2 }}$ through $I_{\text {outZ2 }}$ are the output currents for each gate in RQFP2. In synchronization with $I_{\mathrm{x} 1}$ and $I_{\mathrm{x} 2}$, logic operations are performed with a phase separation of $90^{\circ}$. Figure 3 shows a correct operation of the RFA with $a$ $=1, b=0, c_{\text {in }}=1, s=0, x=1, y=0$, and $c_{\text {out }}=1$.

\subsection{Thermodynamic reversibility}

In order to demonstrate the thermodynamic reversibility of the RFA, we analyzed the time evolution of the phase differences across the Josephson junctions in the RFA because the phase differences are state variables in AQFP logic. Figure 4 shows the evolution of the phase differences across the Josephson junctions for $a=1, b=0$, and $c_{\text {in }}=1$. Here, $I_{\mathrm{x} 1}$ and $I_{\mathrm{x} 2}$ are 1 $\mathrm{GHz}$ excitation currents. For each gate, the phase differences of a pair of Josephson junctions $\left(J_{\mathrm{s} 1}\right.$ and $J_{\mathrm{s} 2}$ for an SPL gate, $J_{\mathrm{m} 1}$ and $J_{\mathrm{m} 2}$ for an MAJ gate in figure 1$)$ are shown. The logic state of each gate is regarded as 1 when the phase difference across $J_{1}$ exceeds $\pi$, whereas it is 0 when the phase difference across $J_{2}$ goes below $-\pi$. Figure 4 shows that all of the phase differences change gradually along with $I_{\mathrm{x} 1}$ and $I_{\mathrm{x} 2}$, which indicates that the RFA can operate quasi-statically, or thermodynamically reversibly [17]. We also confirmed that the phase differences in the RFA change gradually during excitation for other input data combinations. Note that in a thermodynamically irreversible system the phase differences of some Josephson junctions change suddenly, or non-adiabatically, during excitation [19]. 
In order to demonstrate the thermodynamic reversibility of the RFA from the viewpoint of dissipation, we simulated the energy dissipation of the RFA in a similar manner to a previous study [14]. Figure 5 shows the schematic of the circuit used for simulation. Four-stage AQFP buffers are inserted before and after the RFA to avoid the interactions with the input and output ports. Figure 6 shows the simulation results for the energy dissipation per operation of the RFA as a function of the frequency of the excitation current. As a reference, we also simulated the energy dissipation per operation of an AQFP full adder (AQFP FA) [20], where conventional irreversible AQFP logic gates, such as MAJ gates, are used. In general, the energy dissipation of AQFP gates depends on input data combinations [14]. Thus, the energy dissipation shown in figure 6 is the average between the values for all input data combinations. The lines show the simulation results at $T=0 \mathrm{~K}$, and the markers show those at $T=4.2 \mathrm{~K}$. The markers are the averages over 500 iterations. In the simulation at $4.2 \mathrm{~K}$, thermal noise current sources are added in parallel to the Josephson junctions. The amplitude of the thermal noise currents is given by the Monte Carlo method and follows the Gaussian law with the standard deviation given by $\left(2 k_{\mathrm{B}} T / R_{\mathrm{sg}} \Delta t\right)^{0.5}$ [21], where $\Delta t$ is a simulation time step, and $R_{\mathrm{sg}}$ is the sub-gap resistance. In this study, $\Delta t=0.2 \mathrm{ps}, R_{\mathrm{sg}}=200 \Omega$. In figure 6 , there are no remarkable differences between the calculation results at $0 \mathrm{~K}$ and those at $4.2 \mathrm{~K}$. The average energy dissipation of the RFA decreases linearly and approaches zero as the frequency decreases, which indicates that there is no minimum boundary in the energy dissipation for logic operations using the RFA, and the RFA can operate thermodynamically reversibly in the quasi-static limit even at finite temperature. On the other hand, the energy dissipation of the AQFP FA approaches a non-zero value as the frequency decreases. This is because, in a thermodynamically irreversible system, the Josephson junctions that operate non-adiabatically impose non-zero minimum energy bounds [14]. In this simulation, the energy dissipation of the RFA is smaller than that of the 
AQFP FA for the entire frequency range. At $0.01 \mathrm{GHz}$, the energy dissipation of the RFA is even lower than the thermal energy at $4.2 \mathrm{~K}, 5.80 \times 10^{-23} \mathrm{~J}$.

\subsection{Experimental demonstration}

The above simulation results verify the thermodynamic reversibility of the RFA. We next fabricated an RFA to show that RQFP-based logic circuits can be achieved using practical fabrication technology. The RFA was designed and fabricated using the AIST $10 \mathrm{kA} \mathrm{cm}^{-2} \mathrm{Nb}$ high-speed standard process (HSTP) [15]. The physical layout design of the RQFP gates in the RFA was based on designs reported in the literature [16]. Figure 7(a) shows a microphotograph of the RFA, where $I_{\mathrm{x} 1}$ and $I_{\mathrm{x} 2}$ are excitation currents; $a, b$, and $c_{\text {in }}$ are the inputs; and $s, x, y$, and $c_{\text {out }}$ are the outputs, which are read out using dc superconducting quantum interference devices (dc-SQUIDs) [11]. Figure 7(b) shows the waveforms for the RFA at $100 \mathrm{kHz}$ in liquid He. We confirmed the correct logic operations for all input data combinations. The measurement results for the excitation current margins of $I_{\mathrm{x} 1}$ and $I_{\mathrm{x} 2}$ were $5.4 \mathrm{~dB}$ and $6.1 \mathrm{~dB}$, respectively, which indicates that RQFP-based logic circuits can operate stably using practical circuit parameters and fabrication technology.

\section{Conclusion}

We investigated the reversibility and feasibility of the RFA. We confirmed that the phase differences across the Josephson junctions in the RFA change adiabatically, and the energy dissipation of the RFA decreases linearly as the operating frequency decreases. These results indicate that the RFA is a thermodynamically and logically reversible logic circuit. We experimentally demonstrated the RFA, confirming correct operations for all input data combinations. The obtained results suggest that RQFP gates can be used as building blocks to 
design reversible logic circuits.

\section{Acknowledgements}

The present study was supported by a Grant-in-Aid for Scientific Research (S) (Grant No. 26220904) from the Japan Society for the Promotion of Science (JSPS) and PRESTO of the Japan Science and Technology Agency (JST) (Grant No. JPMJPR1528). The devices were fabricated in the clean room for analog-digital superconductivity (CRAVITY) of the National Institute of Advanced Industrial Science and Technology (AIST).

\section{References}

[1] Ball P 2012 Computer engineering: Feeling the heat Nature 492 174-6

[2] Service R F 2012 Computer science. What it'll take to go exascale Science 335 394-6

[3] Fredkin E and Toffoli T 1982 Conservative logic Int. J. Theor. Phys. 21 219-53

[4] Bennett C H 1982 The thermodynamics of computation-a review Int. J. Theor. Phys. 21 $905-40$

[5] Semenov V K, Danilov G V and Averin D V 2007 Classical and Quantum Operation Modes of the Reversible Josephson-Junction Logic Circuits IEEE Trans. Appl. Supercond. $17455-61$

[6] Lambson B, Carlton D and Bokor J 2011 Exploring the thermodynamic limits of computation in integrated systems: magnetic memory, nanomagnetic logic, and the Landauer limit Phys. Rev. Lett. 107010604

[7] Hänninen I, Lu H, Blair E P, Lent C S and Snider G L 2014 Reversible and Adiabatic Computing: Energy-Efficiency Maximized Field-Coupled Nanocomputing: Paradigms, Progress, and Perspectives ed N G Anderson and S Bhanja (Berlin, Heidelberg: Springer Berlin Heidelberg) pp 341-56

[8] Wenzler J-S, Dunn T, Toffoli T and Mohanty P 2014 A nanomechanical Fredkin gate Nano Lett. 14 89-93

[9] Fratto B E and Katz E 2015 Reversible Logic Gates Based on Enzyme-Biocatalyzed Reactions and Realized in Flow Cells: A Modular Approach Chemphyschem 16 1405-15

[10] Takeuchi N, Yamanashi Y and Yoshikawa N 2014 Reversible logic gate using adiabatic superconducting devices Sci. Rep. 46354

[11] Takeuchi N, Ozawa D, Yamanashi Y and Yoshikawa N 2013 An adiabatic quantum flux 
parametron as an ultra-low-power logic device Supercond. Sci. Technol. 26035010

[12] Takeuchi N, Ehara K, Inoue K, Yamanashi Y and Yoshikawa N 2013 Margin and Energy Dissipation of Adiabatic Quantum-Flux-Parametron Logic at Finite Temperature IEEE Trans. Appl. Supercond. 231700304

[13] Hosoya M, Hioe W, Casas J, Kamikawai R, Harada Y, Wada Y, Nakane H, Suda R and Goto E 1991 Quantum flux parametron: a single quantum flux device for Josephson supercomputer IEEE Trans. Appl. Supercond. 177-89

[14] Takeuchi N, Yamanashi Y and Yoshikawa N 2017 Reversibility and energy dissipation in adiabatic superconductor logic Sci. Rep. 775

[15] Takeuchi N, Nagasawa S, China F, Ando T, Hidaka M, Yamanashi Y and Yoshikawa N 2017 Adiabatic quantum-flux-parametron cell library designed using a $10 \mathrm{kA} \mathrm{cm}^{-2}$ niobium fabrication process Supercond. Sci. Technol. 30035002

[16] Takeuchi N, Yamanashi Y and Yoshikawa N 2018 Recent Progress on Reversible Quantum-Flux-Parametron for Superconductor Reversible Computing IEICE Transactions on Electronics E101.C 352-8

[17] Sagawa T 2014 Thermodynamic and logical reversibilities revisited J. Stat. Mech. Theory Exp. 2014 P03025

[18] Fang E S and Van Duzer T 1989 A Josephson Integrated Circuit Simulator (JSIM) for Superconductive Electronics Application Extended Abstracts of Int. Superconductivity Electronics Conf. International Superconductivity Electronics Conference (ISEC) (UC Berkley) pp 407-10

[19] Takeuchi N, Yamanashi Y and Yoshikawa N 2015 Thermodynamic Study of Energy Dissipation in Adiabatic Superconductor Logic Phys. Rev. Applied 4034007

[20] Inoue K, Takeuchi N, Ehara K, Yamanashi Y and Yoshikawa N 2013 Simulation and Experimental Demonstration of Logic Circuits Using an Ultra-Low-Power Adiabatic Quantum-Flux-Parametron IEEE Trans. Appl. Supercond. 231301105

[21] Jeffery M, Xie P Y, Whiteley S R and Van Duzer T 1999 Monte Carlo and thermal noise analysis of ultra-high-speed high temperature superconductor digital circuits IEEE Trans. Appl. Supercond. 9 4095-8 


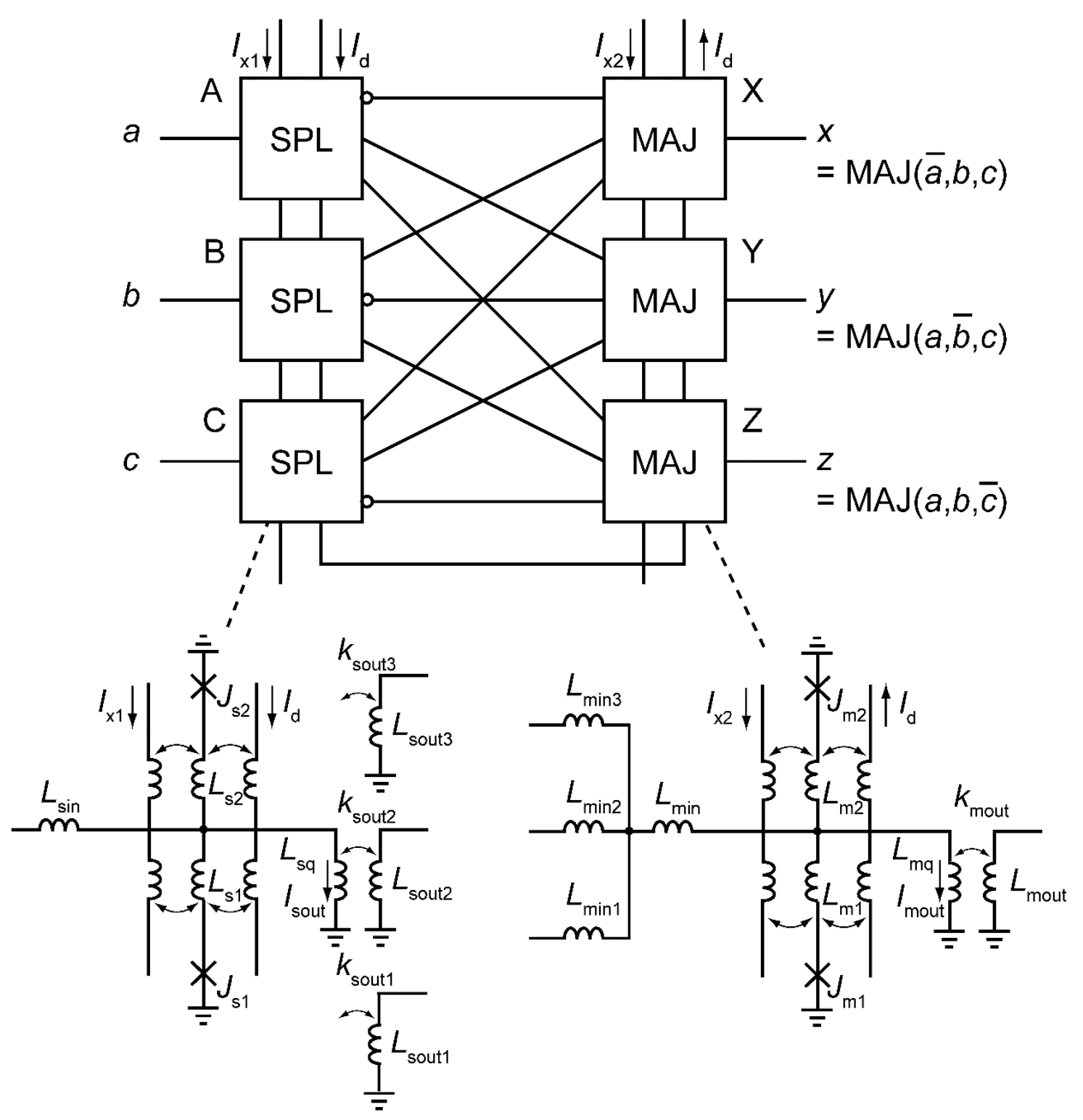

Figure 1. Schematic of the RQFP gate. The gate is composed of six AQFP gates. Three of these gates work as three-input majority (MAJ) gates, and the other three gates work as three-output splitter (SPL) gates. Here, $a, b$, and $c$ are the input data; and $x, y$, and $z$ are the output data. The circuit topology of the RFA gate is symmetrical because the three-input MAJ gate and the three-output SPL gate have the same circuit schematics. Logic negation is achieved by changing the sign of the coupling coefficients $\left(k_{\text {sout1 }}, k_{\text {sout } 2}\right.$, and $\left.k_{\text {sout3 } 3}\right)$. 


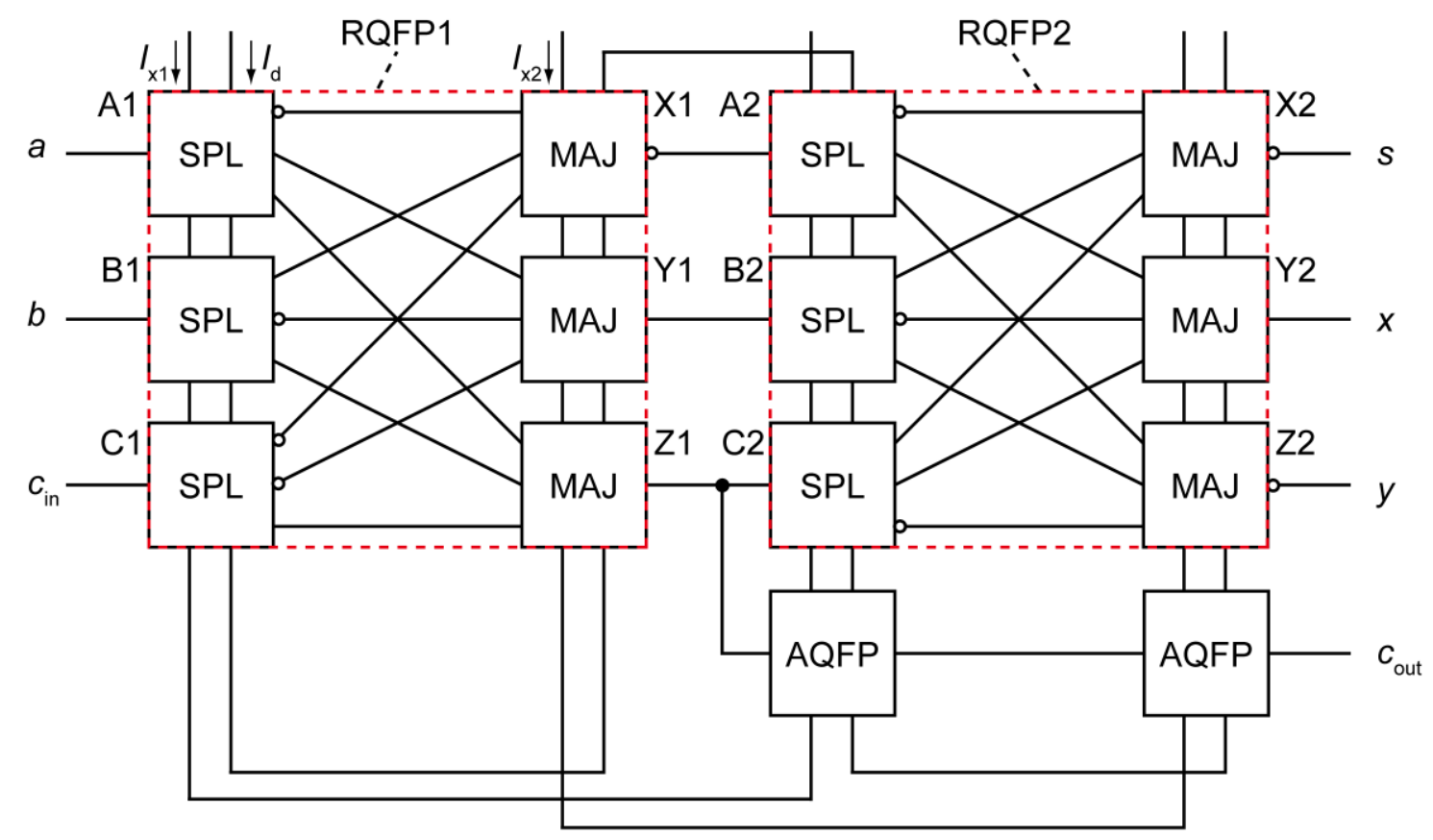

Figure 2. Schematic of the RFA, which is composed of two RQFP gates. Here, $a, b$, and $c_{\text {in }}$ are the input data; and $s$ and $c_{\text {out }}$ represent the sum and carry outputs, respectively. In addition, $x$ and $y$ are garbage outputs, which correspond to $c_{\text {in }}$ and $b$, respectively. 
Table 1. Truth table for the RFA.

\begin{tabular}{ccccccc}
\hline$a$ & $b$ & $c_{\text {in }}$ & $s$ & $x$ & $y$ & $c_{\text {out }}$ \\
\hline 0 & 0 & 0 & 0 & 0 & 0 & 0 \\
0 & 0 & 1 & 1 & 1 & 0 & 0 \\
0 & 1 & 0 & 1 & 0 & 1 & 0 \\
0 & 1 & 1 & 0 & 1 & 1 & 1 \\
1 & 0 & 0 & 1 & 0 & 0 & 0 \\
1 & 0 & 1 & 0 & 1 & 0 & 1 \\
1 & 1 & 0 & 0 & 0 & 1 & 1 \\
1 & 1 & 1 & 1 & 1 & 1 & 1 \\
\hline
\end{tabular}


(arb. unit)

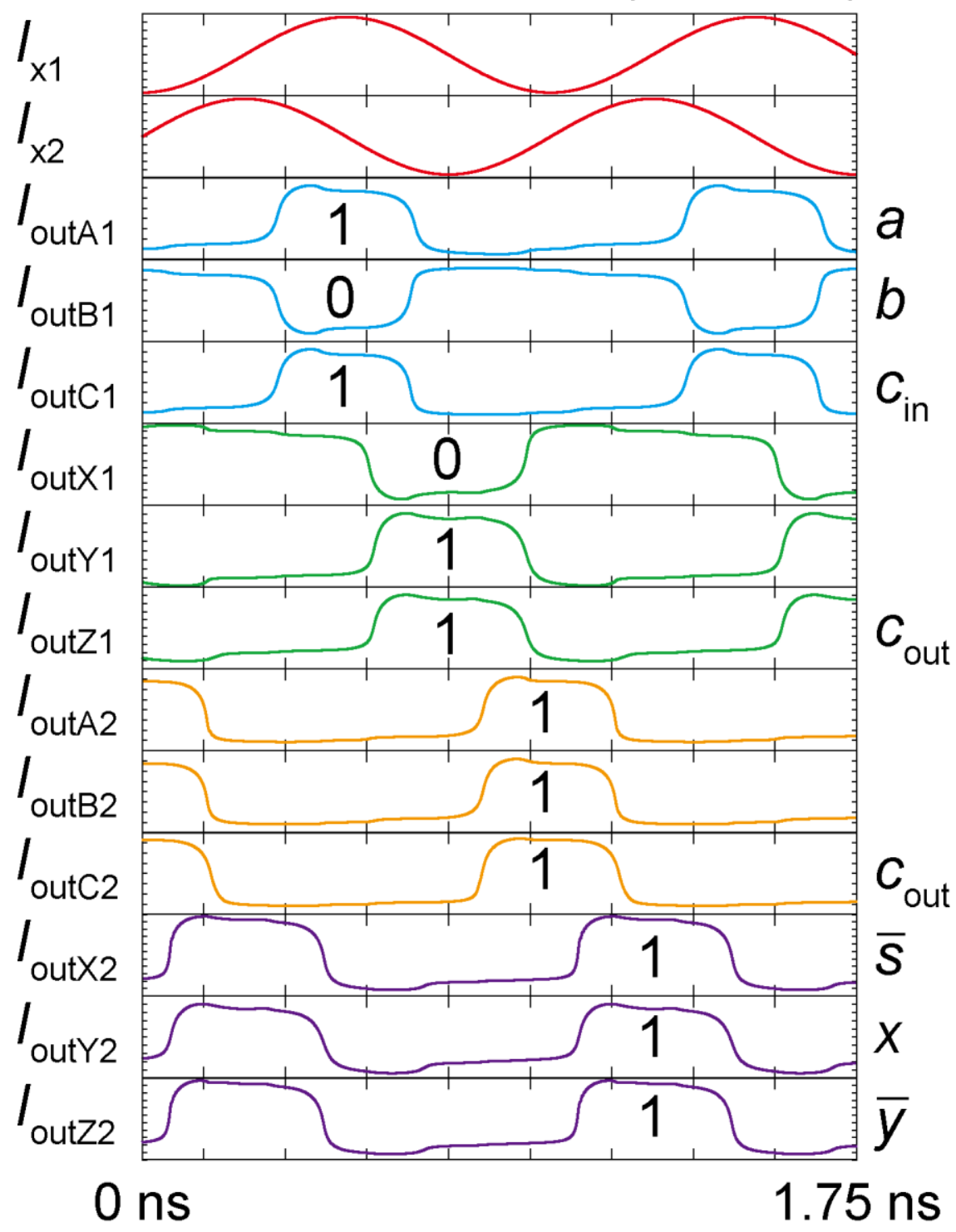

Figure 3. Transient analysis results for the RFA. Logic operations are performed with $1 \mathrm{GHz}$ excitation currents $I_{\mathrm{x} 1}$ and $I_{\mathrm{x} 2}$ with a phase separation of $90^{\circ}$. Here, $I_{\text {outA1 }}$ through $I_{\text {outZ1 }}$ are the output currents for each AQFP gate in RQFP1, and $I_{\text {outA2 }}$ through $I_{\text {outZ2 }}$ are the output currents for each gate in RQFP2. 


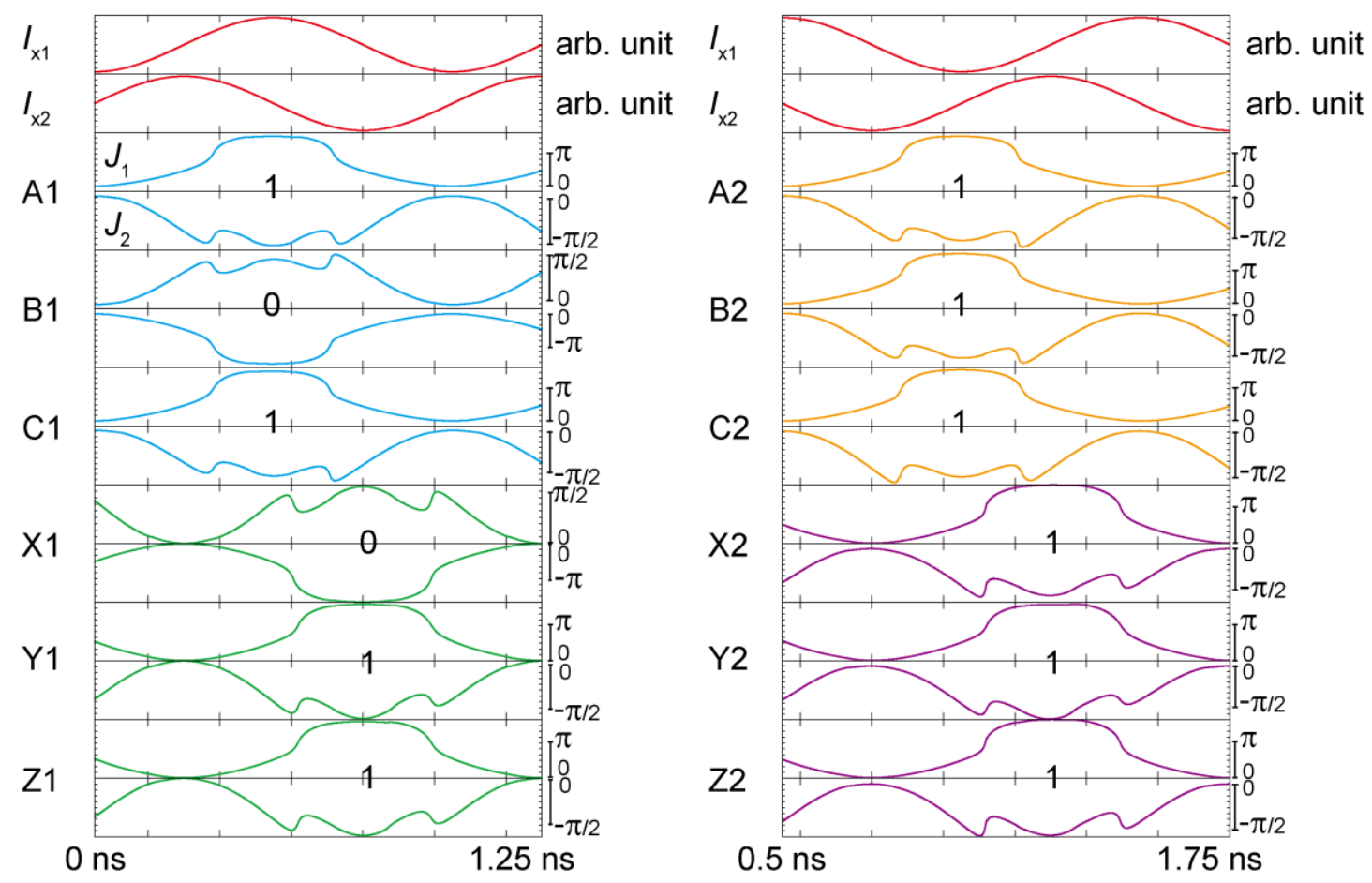

Figure 4. Time evolution of the phase differences across the Josephson junctions in the RFA for $\left(a, b, c_{\text {in }}\right)=(1,0,1)$. The phase differences change gradually along with $I_{\mathrm{x} 1}$ and $I_{\mathrm{x} 2}$, which indicates that the RFA can operate quasi-statically, or thermodynamically reversibly. 


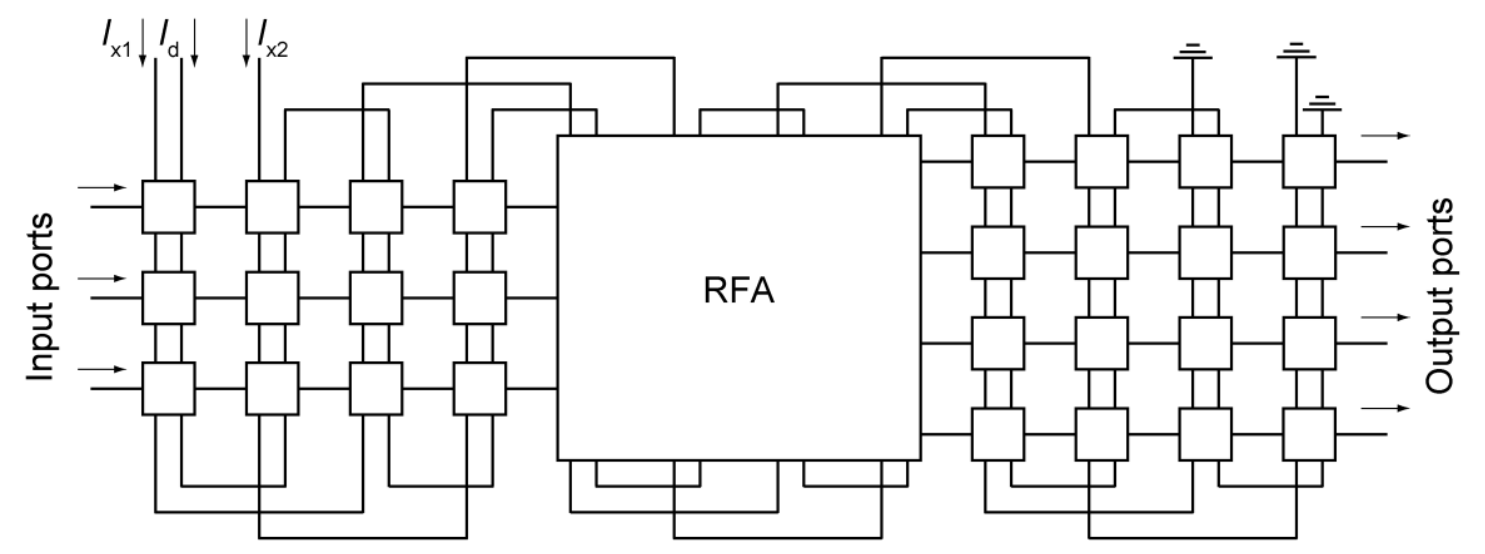

Figure 5. Schematic of the circuit used for simulation. Four-stage AQFP buffers are inserted before and after the RFA to avoid the interactions with the input and output ports. $I_{\mathrm{x} 1}$ and $I_{\mathrm{x} 2}$ represent excitation currents; and $I_{\mathrm{d}}$ is a dc-offset current. 


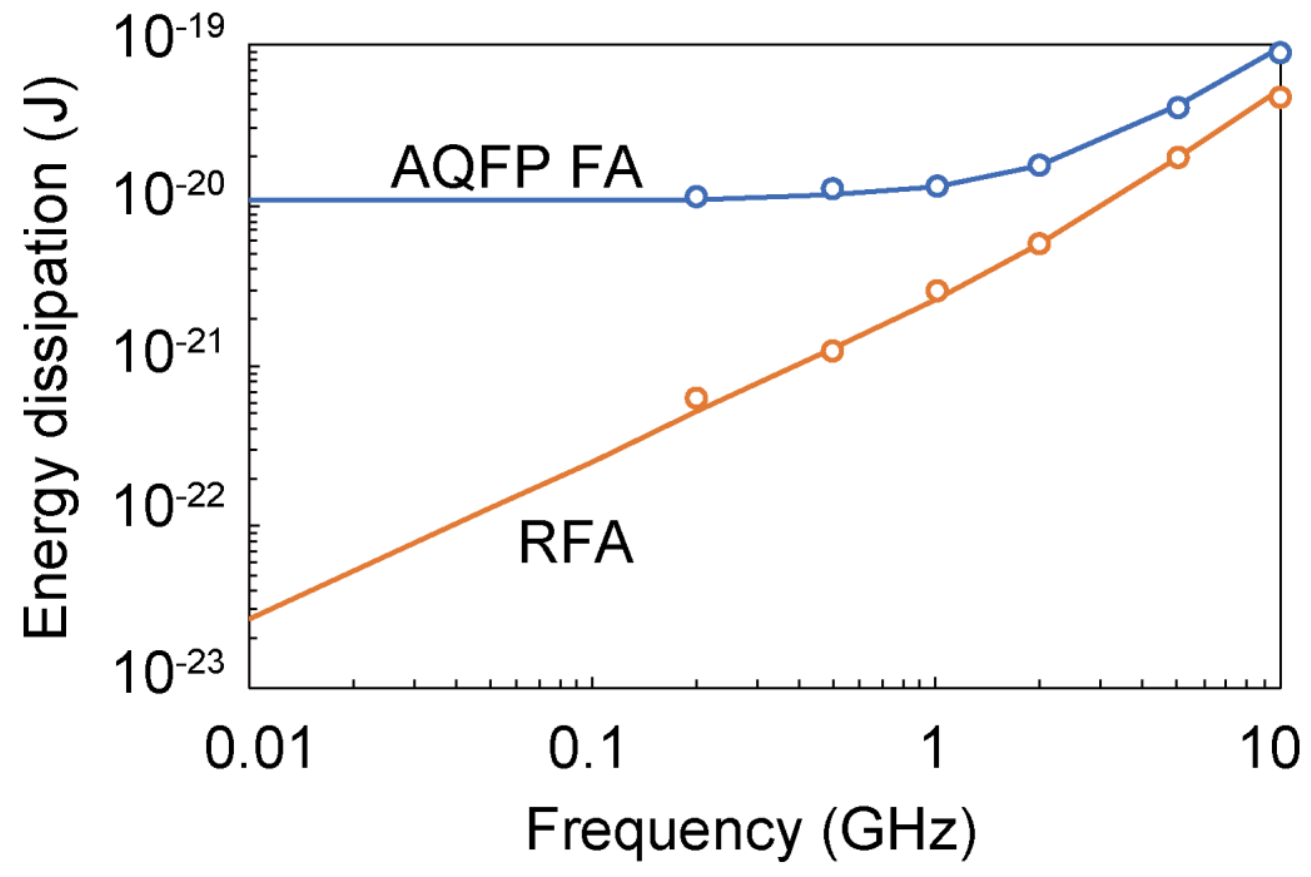

Figure 6. Simulation results for the energy dissipation of the RFA and AQFP FA as a function of the frequency of the excitation current. The lines show the simulation results at $T=0 \mathrm{~K}$ and the markers show those at $T=4.2 \mathrm{~K}$, which are the averages over 500 iterations. In the simulation, the energy dissipation is averaged between the values for all input data combinations. The energy dissipation for the RFA decreases linearly and approaches zero as the frequency decreases, which indicates that the RFA can operate thermodynamically reversibly in the quasi-static limit. On the other hand, the energy dissipation for the AQFP FA approaches a non-zero value. 


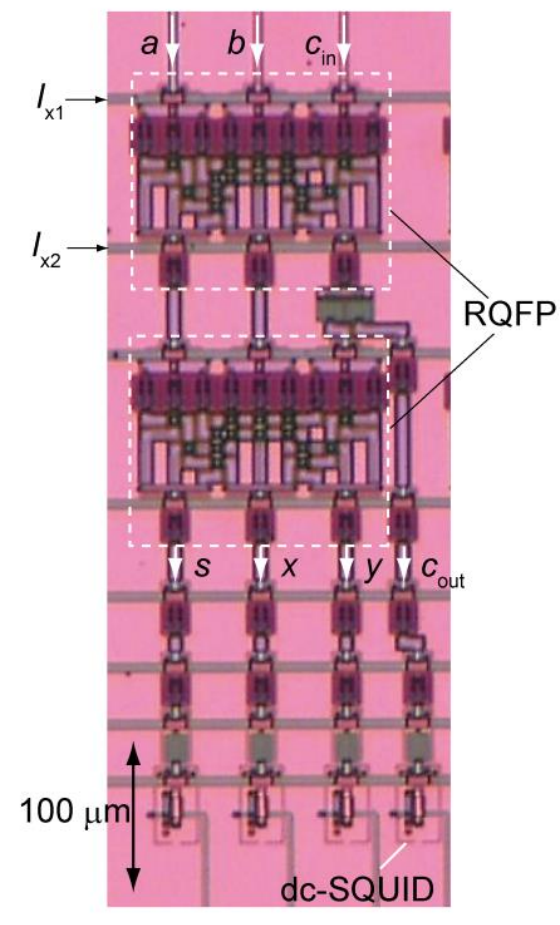

(a)

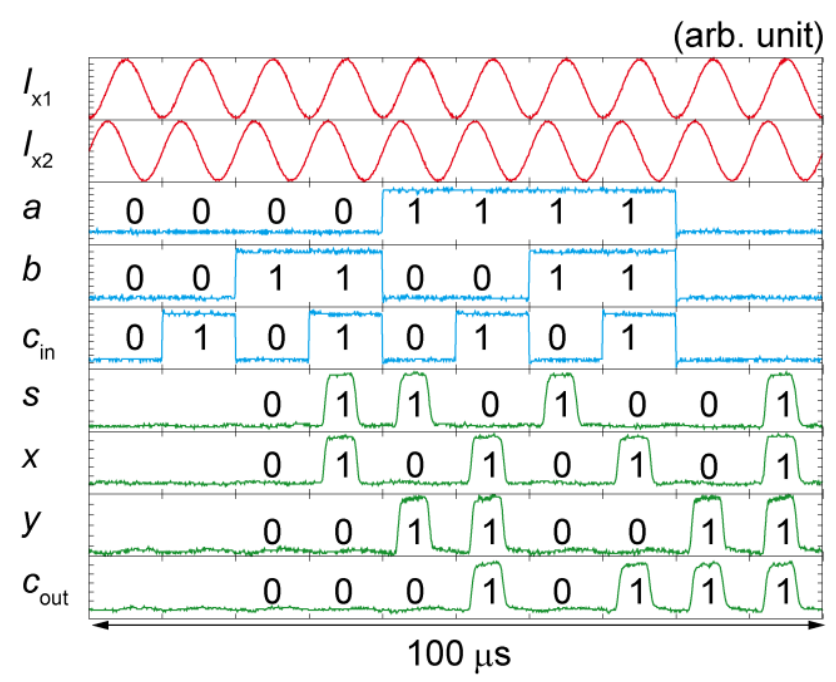

(b)

Figure 7. Experimental demonstration of the RFA. (a) Micrograph of the RFA. The outputs are read out through dc-SQUIDs. (b) Measurement results for the RFA at $100 \mathrm{kHz}$ at $4.2 \mathrm{~K}$. Here, $I_{\mathrm{x} 1}$ and $I_{\mathrm{x} 2}$ represent excitation currents; $a, b$, and $c_{\text {in }}$ are the inputs; and $s, x, y$, and $c_{\text {out }}$ are the outputs. Correct operation of the RFA is confirmed for all input data combinations. 